\title{
A COMPUTATIONAL PROCEDURE FOR LARGE ROTATIONAL MOTIONS IN MULTIBODY DYNAMICS
}

by

K. C. PARK and J. C. CHIOU

COLLEGE OF ENGINEERING UNIVERSITY OF COLORADO BOULDER, COLORADO 80309

(NASA-Ch-18122.2) FOR LAEGE BOTATIONAL MOTIONS IN MULTIBODY

N $87-30111$ DYNAMICS Semiannud 1 status keport, 13 Apr. 13 uct. 1987 (Colorado Univ.) $28 \mathrm{p}$ Avail: NTIS HC AO3/MF AO 1 CSCE $12 \AA \quad$ G3/64 
A Computational Procedure for Large Rotational Motions in Multibody Dynamics

K. C. Park and J. C. Chiow ${ }^{2}$

Center for Space Structures and Controls

University of Colorado. Campus Box 427

Boulder, Colorado 80309

August 1987

Report No. CU-CSSC-87-08

Research Sponsored by NASA/Langley Research Center

Grant No. NAG - 1 - 756

${ }^{1}$ Professor of Aerospace Engineering Sciences and Mechanical Engineering. Member of AIAA.

${ }^{2}$ Graduate Research Assistant. Department of Mechanical Engineering. 


\begin{abstract}
A computational procedure suitable for the solution of equations of motion for multibody systems is presented. The present procedure adopts a differential partitioning of the translational motions and the rotational motions. The translational equations of motion are then treated by either a conventional explicit or an implicit direct integration method. A principal feature of the present procedure is a nonlinearly implicit algorithm for updating rotations via the Euler four-parameter representation. The present procedure is applied to the rolling of a sphere through a specified trajectory. which indicates that the procedure yields robust solutions.
\end{abstract}




\section{Introduction}

The numerical solution of the equations of motion for multibody systems has been continuously challenging the dynamist. In general, computer simulation of multibody dynamical (MBD) systems requires a concerted integration of several computational aspects. These include a data structure for describing the system topology, computerized generation of the governing equations of motion, incorporation and accurate treatment of constraint conditions, implementation of suitable solution algorithms and easy interpretation of the simulation results.

In the past, the task of formulating equations of motion has been a dominant concern to many dynamists (see, e.g., [1-6]). From the computational viewpoint, it can be said that the differences in existing formulations lie principally in their ways of incorporating constraints $[7-15]$ and in their resulting system topologies $[2,16,17]$. When the MBD systems become more complex, such as in the deployment of large space structures, streamlined accommodation of system topologies becomes a more important concern than elegance of formulation. This is because a flexible data structure can allow different modeling, different formulations and different solution techniques to be adapted to different subsystems. In a previous study [14-15], a new constraint treatment technique that can solve the constraint equations in a separate module from that for the translation and rotation variables was presented. A major feature of that study was to preserve the system topology for a variety of MBD systems.

In order to provide a complete set of solution modules, the constraint solution module must be interfaced with a solution module for the primary variables, viz, the translational and rotational variables. It is generally agreed that the solution of the translational motions can be treated either by a conventional explicit or an implicit direct integration method.

However, a wide spectrum of solution techniques has been proposed to integrate the rotational motions. Yet, along with constraint algorithms, many solution reliability and 
efficiency issues in multibody simulation packages appear to hinge on how one solves the rotational degrees of freedom. This is especially true for flexible multibody systems wherein the higher frequency-response components are often due to rotational ocillatory motions. The objective of the present paper is thus to present a computational procedure for a robust and efficient treatment of rotational motions so that one can solve general MBD equations for a variety of system topologies.

\section{Equations of Motion for Multibody Systems}

The discrete equations of motion for flexible multibody systems can be expressed as [16]:

$$
\begin{array}{lr}
M \ddot{\mathbf{u}}+D(\dot{\mathbf{u}})+\mathbf{S}(u)+\mathbf{B}_{N}^{T} \lambda_{N}+\mathbf{B}_{H}^{T} \lambda_{H}=\mathbf{f}(t) \\
\boldsymbol{\Phi}_{N}(\dot{\mathbf{u}}, t)=0, & \boldsymbol{\Phi}_{H}(\mathbf{u}, t)=0
\end{array}
$$

where $M$ is the mass matrix, $D(\cdot)$ is the generalized velocity-dependent force operator, $S(\cdot)$ is the internal force operator due to member flexibility, $B_{N}$ and $B_{H}$ are the gradients of the nonholonomic and holonomic constraints (2.2), $\lambda_{N}$ and $\lambda_{H}$ are the constraint forces, $f(t)$ is the applied force, $\mathbf{u}$ is the generalized displacement vector, $\left({ }^{\circ}\right)$ denotes time differentiation and ()$^{T}$ designates the matrix transposition.

The solution of (2.1) and (2.2) consists of two tasks: the satisfaction of the constraint conditions (2.2) to obtain $\lambda$ and the computation of $u$ from (2.1). Procedures to obtain $\lambda_{N}$ and $\lambda_{H}$ by satisfying $(2.2)$ were presented in $[14,15]$ and will be adopted in Numerical Experiments. Hence, we will concentrate on the computation of $\mathbf{u}$.

\section{Differential Partitioning of the MBD Equations}

A basic difficulty in direct integration of (2.1) is that $\dot{\omega}$ and $\omega$ are not directly integrable, except for some special kinematic configurations. This motivates us to partition $\tilde{\mathbf{u}}$ into the 
translational acceleration vector, $\tilde{d}$, which is directly integrable and the angular acceleration vector, $\dot{\omega}$, which is not, and treat them by a partitioned solution procedure [18-20], viz

$$
\ddot{\mathrm{u}}=\left\{\begin{array}{c}
\ddot{\mathrm{d}} \\
\dot{\boldsymbol{\omega}}
\end{array}\right\}, \quad \dot{\mathrm{u}}=\left\{\begin{array}{c}
\dot{\mathrm{d}} \\
\omega
\end{array}\right\}
$$

The equations of motion (2.1) can be partitioned according to the above acceleration partitioning:

$$
\left[\begin{array}{cc}
M_{d} & 0 \\
0 & M_{\omega}
\end{array}\right]\left\{\begin{array}{l}
\dot{\mathrm{d}} \\
\dot{\omega}
\end{array}\right\}+\left\{\begin{array}{l}
Q_{d} \\
Q_{\omega}
\end{array}\right\}=\left\{\begin{array}{l}
f_{d} \\
f_{\omega}
\end{array}\right\}
$$

where

$$
\left\{\begin{array}{l}
Q_{d} \\
Q_{\omega}
\end{array}\right\}=\left\{\begin{array}{l}
\mathbf{D}_{d}(\dot{\mathbf{u}})+\mathbf{S}_{d}(\mathbf{u})-B_{d}^{T} \lambda \\
\mathbf{D}_{\omega}(\dot{\mathbf{u}})+\mathbf{S}_{\omega}(\mathbf{u})-B_{\omega}^{T} \boldsymbol{\lambda}
\end{array}\right\}
$$

in which

$$
B=B_{N}+B_{H}, \quad \lambda=\lambda_{N}+\lambda_{H}
$$

To effect the node-by-node integration for the rotational degrees of freedom, we partition $\dot{\omega}$ further into

$$
\dot{\omega}=\left\lfloor\dot{\omega}^{1}, \dot{\omega}^{2}, \ldots, \dot{\omega}^{P}\right\rfloor^{T}
$$

where $\dot{\omega}^{(j)}$ is a $(3 \times 1)$ angular acceleration vector for the $j$-th node,

$$
\dot{\omega}^{(j)}=\left\lfloor\omega_{1}^{(j)}, \omega_{2}^{(j)}, \omega_{3}^{(j)}\right\rfloor
$$


4. Review of Staggered Stabilized Procedure for Constraints

For simplicity, we rewrite (2.1) and (2.2) as

$$
\begin{gathered}
M \ddot{\mathbf{u}}+D(\dot{\mathbf{u}})+S(\mathbf{u})+B^{T} \lambda=\mathbf{f} \\
\Phi(\mathbf{u}, \quad \dot{\mathbf{u}}, \quad t)=0
\end{gathered}
$$

A penalty technique is introduced to obtain the constraints as follows

$$
\lambda=\frac{1}{\epsilon} \Phi(\mathrm{u}, \quad \dot{\mathrm{u}}, \quad t), \quad 0<\epsilon<<1
$$

Differentiation of $\lambda$ with respect to time yields

$$
\epsilon \dot{\lambda}=B \dot{\mathbf{u}}+\dot{B} \dot{u}+\frac{\partial \Phi}{\partial t}
$$

Substituting $\overline{\mathrm{u}}$ from (4.1) into (4.4), we obtain

$$
\epsilon \dot{\lambda}+B M^{-1} B^{T} \lambda=B M^{-1}(\mathbf{f}-D(\dot{\mathbf{u}})-S(\mathbf{u}))+\dot{B} \dot{\mathbf{u}}+\frac{\partial \Phi}{\partial t}=\mathbf{r}_{\lambda}
$$

Hence, the solution of (4.1) and (4.2) has been replaced by (3.2) and (4.5).

\section{Nonlinearly Implicit Procedure for Large Rotations}

\subsection{Euler Parameters and Angular Accelerations}

The four-parameter Euler representation of the angular velocity for each node is expressed as (see, e.g., Wittenburg[21]):

$$
\dot{\mathbf{q}}=\frac{1}{2}\left[\begin{array}{cc}
0 & -\omega^{T} \\
\omega & -\tilde{\omega}
\end{array}\right] \mathbf{q}=A(\omega) \mathbf{q}, \quad \mathbf{q}=\left[\begin{array}{llll}
q_{0} & q_{1} & q_{2} & q_{3}
\end{array}\right]^{T}
$$

where

$$
\tilde{\omega}=\left[\begin{array}{ccc}
0 & -\omega_{3} & \omega_{2} \\
\omega_{3} & 0 & -\omega_{1} \\
-\omega_{2} & \omega_{1} & 0
\end{array}\right], \quad \omega=\left[\begin{array}{lll}
\omega_{1} & \omega_{2} & \omega_{3}
\end{array}\right]
$$


and the nodal-designating superscript is omitted for notational simplicity.

Time differentiation of (5.1) once more yields

$$
\ddot{\mathbf{q}}=\mathbf{A}(\dot{\omega}) \cdot \mathbf{q}+\mathbf{A}(\omega) \cdot \dot{\mathbf{q}}
$$

where $\mathbf{A}(\dot{\omega})$ is obtained by substituting $\dot{\omega}$ for $\omega$ in $\mathbf{A}(\omega)$.

Note that (5.3) contains the constraint condition

$$
q^{T} q=1
$$

in its second-derivative form:

$$
\mathbf{q}^{T} \ddot{\mathbf{q}}+\dot{\mathbf{q}}^{T} \dot{\mathbf{q}}=0
$$

\subsection{Mid-Point Integration of Euler Parameters}

Suppose that we know the state variables, $\dot{\omega}^{k}$ and $\omega^{k}$, at the k-th time step and we want to solve for $\mathrm{q}^{k+1}$. Because of the special properties of $A(\omega)$ and $A(\dot{\omega})$, one can take advantage of the following set of mid-point rules:

$$
\left\{\begin{array}{l}
\mathbf{q}^{k+\frac{1}{2}}=\mathbf{q}^{k}+\delta \dot{\mathbf{q}}^{k+\frac{1}{2}} \\
\dot{\mathbf{q}}^{k+\frac{1}{2}}=\dot{\mathbf{q}}^{k}+\delta \ddot{\mathbf{q}}^{k+\frac{1}{2}} \\
\mathbf{q}^{k+1}=2 \mathbf{q}^{k+\frac{1}{2}}-\mathbf{q}^{k}, \quad \delta=\frac{h}{2}
\end{array}\right.
$$

where $h$ is the stepsize.

Substituting (5.1) and (5.3) into (5.6), one obtains

$$
\left\{\begin{array}{l}
{\left[I-\delta \mathbf{A}\left(\omega^{k+\frac{1}{2}}\right)-\delta^{2} \mathbf{A}\left(\dot{\omega}^{k+\frac{1}{2}}\right)\right] \mathbf{q}^{k+\frac{1}{2}}=\left[I-\delta \mathbf{A}\left(\omega^{k+\frac{1}{2}}\right)+\delta \mathbf{A}\left(\omega^{k}\right)\right] \mathbf{q}^{k}} \\
{\left[I-\delta \mathbf{A}\left(\omega^{k+\frac{1}{2}}\right)\right] \dot{\mathbf{q}}^{k+\frac{1}{2}}=\dot{\mathbf{q}}^{k}+\delta \mathbf{A}\left(\omega^{k+\frac{1}{2}}\right) \mathbf{q}^{k+\frac{1}{2}}, \quad\left(\mathbf{q}^{k+\frac{1}{2}}\right)^{T} \dot{\mathbf{q}}^{k+\frac{1}{2}}=0}
\end{array}\right.
$$

Since $\mathbf{A}\left(\omega^{k+\frac{1}{2}}\right)$ and $\mathbf{A}\left(\dot{\omega}^{k+\frac{1}{2}}\right)$ are not available, we approximate them by

$$
\mathbf{A}\left(\omega^{k+\frac{1}{2}}\right) \simeq \mathbf{A}\left(\omega^{k}\right) \quad, \quad \mathbf{A}\left(\dot{\omega}^{k+\frac{1}{2}}\right) \simeq \mathbf{A}\left(\dot{\omega}^{k}\right)
$$


which are equivalent to a Newton-like tangent approximation. Hence, we have from (5.7a) and (5.8)

$$
\left[I-\delta \mathbf{A}\left(\omega^{k}\right)-\delta^{2} \mathbf{A}\left(\dot{\omega}^{k}\right)\right] \cdot \mathbf{q}^{k+\frac{1}{2}}=\mathbf{q}^{k}
$$

Once $\mathrm{q}^{k+\frac{1}{2}}$ is obtained from (5.9), we can obtain $\dot{\mathrm{q}}^{k+\frac{1}{2}}$ from (5.7b) by

$$
\left[I-\delta \mathbf{A}\left(\omega^{k+\frac{1}{2}}\right)\right] \dot{\mathbf{q}}^{k+\frac{1}{2}}=\dot{\mathbf{q}}^{k}+\delta \mathbf{A}\left(\omega^{k+\frac{1}{2}}\right) \mathbf{q}^{k+\frac{1}{2}}
$$

Finally, one can update $\omega$ from the following formula:

$$
\boldsymbol{\omega}=2\left(q_{0} \dot{\mathbf{g}}-\tilde{\mathbf{g}} \dot{\mathbf{g}}-\dot{q}_{0} \mathbf{g}\right), \quad \mathbf{g}=\left\lfloor\begin{array}{lll}
q_{1} & q_{2} & q_{3}
\end{array}\right\rfloor, \quad q_{0}^{2}+\mathbf{g}^{T} \mathbf{g}=1
$$

where $\tilde{\mathbf{g}}$ has the same form as $\tilde{\boldsymbol{\omega}}$ as given by (5.2). Hence, if necessary, one can obtain $\dot{\boldsymbol{\omega}}$ from the equations of motion and iterate on $q$ and $\dot{q}$ by (5.7).

The skew-symmetric solution matrices in the above difference equations can be explicitiy inverted via the formula:

$$
\left[\begin{array}{cccc}
1 & -a & -b & -c \\
a & 1 & c & -b \\
b & -c & 1 & a \\
c & b & -a & 1
\end{array}\right]^{-1}=\frac{1}{1+a^{2}+b^{2}+c^{2}}\left[\begin{array}{cccc}
1 & a & b & c \\
-a & 1 & -c & b \\
-b & c & 1 & -a \\
-c & -b & a & 1
\end{array}\right]
$$

Hence, the solution of (5.9) and (5.10) becomes straightforward.

\subsection{Update of New Angular Orientation}

Once $\mathrm{q}^{k+1}$ is computed from (5.9) and (5.6c), it is often required to compute the body-fixed basis vector, $b=\left[\begin{array}{lll}b_{1} & b_{2} & b_{3}\end{array}\right]^{T}$ in terms of the inertial basis vectors, $e=\left[\begin{array}{lll}e_{1} & e_{2} & e_{3}\end{array}\right]^{T}$. These two vectors are related by

$$
\mathbf{b}=\mathbf{R e}
$$

where

$$
\mathbf{R}=\left[\begin{array}{lll}
2\left(q_{0}^{2}+q_{1}^{2}\right)-1 & 2\left(q_{1} q_{2}+q_{0} q_{3}\right) & 2\left(q_{1} q_{3}-q_{0} q_{2}\right) \\
2\left(q_{1} q_{2}-q_{0} q_{3}\right) & 2\left(q_{0}^{2}+q_{2}^{2}\right)-1 & 2\left(q_{2} q_{3}+q_{0} q_{1}\right) \\
2\left(q_{1} q_{3}+q_{0} q_{2}\right) & 2\left(q_{2} q_{3}-q_{0} q_{1}\right) & 2\left(q_{0}^{2}+q_{3}^{2}\right)-1
\end{array}\right]
$$


In order to satisfy the orthonormality of $\mathbf{R}$, it is crucial to satisfy the two constraints, viz, $\mathbf{q}^{T} \mathbf{q}=1$ in computing $\mathbf{q}$ and $\mathbf{q}^{T} \dot{\mathbf{q}}=0$ in computing $\dot{\mathrm{q}}$, respectively. This can be accomplished for $\mathbf{q}^{k+\frac{1}{2}}$ by augmenting the constraint and solving the following equation by a Newton-like procedure:

$$
\left[\begin{array}{cc}
\mathbf{E}_{\mathbf{q}} & \mathbf{q} \\
\mathbf{q}^{T} & 0
\end{array}\right]\left\{\begin{array}{l}
\mathbf{q} \\
\lambda
\end{array}\right\}=\left\{\begin{array}{c}
\mathbf{b}_{q} \\
1
\end{array}\right\}
$$

where $\mathrm{E}_{q}$ is the $(4 \times 4)$ solution matrix in the lefthand side of (5.7a) and $\mathrm{b}_{q}$ is the righthand side vector of $(5.7 \mathrm{a})$, respectively.

Similarly, the constraint $\mathbf{q}^{T} \dot{\mathbf{q}}=0$ can be satisfied by

$$
\left[\begin{array}{cc}
\mathbf{E}_{\dot{q}} & \mathbf{q} \\
\mathbf{q}^{T} & 0
\end{array}\right]\left\{\begin{array}{c}
\dot{\mathbf{q}} \\
\lambda
\end{array}\right\}=\left\{\begin{array}{c}
\mathbf{b}_{\dot{q}} \\
0
\end{array}\right\}
$$

where $E_{\dot{q}}$ is the $(4 \times 4)$ solution matrix in the lefthand side of $(5.10)$ and $b_{\dot{q}}$ is the righthand side vector of $(5.10)$, respectively.

In addition, after the solution has converged at the $\left(k+\frac{1}{2}\right)$-timestep, we must enforce the same two constraint conditions in updating $\mathrm{q}^{k+1}$ and $\dot{\mathrm{q}}^{k+1}$. This is effected by the following simple procedures.

To maintain the constraint $\mathbf{q}^{T} \mathbf{q}=1$ at the new $(k+1)$-th timestep, we employ

$$
\left[\begin{array}{cc}
\mathbf{I} & \mathbf{q} \\
\mathbf{q}^{T} & 0
\end{array}\right]\left\{\begin{array}{l}
\mathbf{q} \\
\lambda
\end{array}\right\}=\left\{\begin{array}{c}
2 \mathbf{q}^{k+\frac{1}{2}}-\mathbf{q}^{k} \\
1
\end{array}\right\}
$$

where $I$ is $(4 \times 4)$ identity matrix. Similarly, to enforce $q^{T} \dot{q}=0$, we use

$$
\left[\begin{array}{cc}
\mathbf{I} & \mathbf{q} \\
\mathbf{q}^{T} & 0
\end{array}\right]\left\{\begin{array}{l}
\dot{\mathbf{q}} \\
\lambda
\end{array}\right\}=\left\{\begin{array}{c}
2 \dot{\mathbf{q}}^{k+\frac{1}{2}}-\dot{\mathbf{q}}^{k} \\
0
\end{array}\right\}
$$

This completes the present nonlinearly implicit procedure for large rotational motions. 


\section{Solution Procedures for MBD Equations}

We recall (3.2) and (4.5) to summarize the present overall computational procedures

$$
\begin{gathered}
M_{d} \dot{\mathrm{d}}=\mathbf{f}_{d}-D_{d}(\dot{\mathrm{u}})-S_{d}(\mathbf{u})-B_{d}^{T} \lambda \\
M_{\omega} \dot{\boldsymbol{\omega}}=\mathbf{f}_{\omega}-D_{\omega}(\dot{\mathrm{u}})-S_{\omega}(\mathbf{u})-B_{\omega}^{T} \lambda \\
\epsilon \dot{\lambda}+B M^{-1} B^{T} \lambda=\mathbf{r}_{\lambda}=B M^{-1}(\mathbf{f}-D(\dot{\mathrm{u}})-S(\mathbf{u}))+\dot{B} \dot{\mathbf{u}}+\frac{\partial \Phi}{\partial t}
\end{gathered}
$$

The computational sequences are as follows.

$$
\begin{gathered}
\left\{\begin{array}{l}
\dot{\mathrm{d}}^{n+\frac{1}{2}}=\mathrm{d}^{n-\frac{1}{2}}+h \dot{\mathrm{d}}^{n} \\
\mathbf{d}^{n+1}=\mathrm{d}^{n}+h \dot{\mathrm{d}}^{n+\frac{1}{2}}
\end{array}\right. \\
\left\{\begin{array}{l}
\mathbf{A}\left(\omega^{k+\frac{1}{2}}\right) \simeq \mathbf{A}\left(\omega^{k}\right) \quad \mathbf{A}\left(\dot{\omega}^{k+\frac{1}{2}}\right) \simeq \mathbf{A}\left(\dot{\omega}^{k}\right) \\
{\left[I-\delta \mathbf{A}\left(\omega^{k+\frac{1}{2}}\right)-\delta^{2} \mathbf{A}\left(\dot{\omega}^{k+\frac{1}{2}}\right)\right] \mathbf{q}^{k+\frac{1}{2}}=\left[I-\delta \mathbf{A}\left(\omega^{k+\frac{1}{2}}\right)+\delta \mathbf{A}\left(\omega^{k}\right)\right] \mathbf{q}^{k}} \\
{\left[I-\delta \mathbf{A}\left(\omega^{k+\frac{1}{2}}\right)\right] \dot{\mathbf{q}}^{k+\frac{1}{2}}=\dot{\mathbf{q}}^{k}+\delta \mathbf{A}\left(\omega^{k+\frac{1}{2}}\right) \mathbf{q}^{k+\frac{1}{2}}, \quad\left(\mathbf{q}^{k+\frac{1}{2}}\right)^{T} \dot{\mathbf{q}}^{k+\frac{1}{2}}=0} \\
\mathbf{q}^{k+1}=2 \mathbf{q}^{k+\frac{1}{2}}-\mathbf{q}^{k} \\
\dot{\mathbf{q}}^{k+1}=2 \dot{\mathbf{q}}^{k+\frac{1}{2}}-\dot{\mathbf{q}}^{k}, \quad\left(\mathbf{q}^{k+1}\right)^{T} \dot{\mathbf{q}}^{k+1}=0 \\
\boldsymbol{\omega}=2\left(q_{0} \dot{\mathbf{g}}-\tilde{\mathbf{g}} \dot{\mathbf{g}}-\dot{\mathbf{q}} 0 \mathbf{g}\right)
\end{array}\right.
\end{gathered}
$$

It should be mentioned that the above procedures require only $(4 \times 4)$-matrices which can be inverted explicitly via (5.12).

$$
\left(\epsilon \mathrm{I}+\delta \mathrm{BM}^{-1} \mathrm{~B}^{T}\right) \lambda^{k+\frac{1}{2}}=\delta \mathbf{r}_{\lambda}^{k+\frac{1}{2}}+\epsilon \lambda^{k}
$$

We will now apply (6.4) $-(6.6)$ together with $(5.15)-(5.18)$ to some sample problems.

\section{An Example-Dynamics of a Bowling Ball}

This problem was investigated by Huston et al [22], whose equations do not involve the constraint force, $\lambda$. In the present analysis, we employ a formulation that incorporates the 
constraint force as part of the system variables. Fig. 1 illustrates the ball with its radius $a$ and an offset center $r_{0}$ that is to follow a sine curve

$$
y=\sin x
$$

The various matrices and vector quantities for (6.1)-(6.3) can be derived as

$$
\begin{aligned}
& \mathbf{M}=\left[\begin{array}{ccccc}
m & 0 & -m r_{0} \mathbf{e}_{1} \cdot \mathbf{b}_{2} & m r_{0} \mathbf{e}_{1} \cdot \mathbf{b}_{1} & 0 \\
0 & m & -m r_{0} \mathbf{e}_{2} \cdot \mathbf{b}_{2} & m r_{0} \mathbf{e}_{2} \cdot \mathbf{b}_{1} & 0 \\
& & J_{1} & 0 & 0 \\
& \text { sym. } & & J_{2} & 0 \\
& & & & J_{3}
\end{array}\right] \\
& B=\left[\begin{array}{ccccc}
1 & 0 & -a b_{1} \cdot \mathbf{e}_{2} & -a b_{2} \cdot \mathbf{e}_{2} & -a b_{3} \cdot \mathbf{e}_{2} \\
0 & 1 & a b_{1} \cdot \mathbf{e}_{1} & a b_{2} \cdot \mathbf{e}_{1} & a b_{3} \cdot \mathbf{e}_{1} \\
\cos x & -1 & 0 & 0 & 0
\end{array}\right] \\
& \mathbf{D}_{d}(\dot{\mathbf{u}})=-m r_{0}\left\{\begin{array}{l}
\omega_{1} \omega_{3} \mathbf{e}_{1} \cdot \mathbf{b}_{1}+\omega_{2} \omega_{3} \mathbf{e}_{1} \cdot \mathbf{b}_{2}-\left(\omega_{1}^{2}+\omega_{2}^{2}\right) \mathbf{e}_{1} \cdot \mathbf{b}_{3} \\
\omega_{1} \omega_{3} \mathbf{e}_{2} \cdot \mathbf{b}_{1}+\omega_{2} \omega_{3} \mathbf{e}_{2} \cdot \mathbf{b}_{2}-\left(\omega_{1}^{2}+\omega_{2}^{2}\right) \mathbf{e}_{2} \cdot \mathbf{b}_{3}
\end{array}\right\} \\
& \mathbf{D}_{\omega}(\dot{\mathrm{u}})=-\left\{\begin{array}{l}
\omega_{2} \omega_{3}\left(J_{2}-J_{3}\right) \\
\omega_{3} \omega_{1}\left(J_{3}-J_{1}\right) \\
\omega_{1} \omega_{2}\left(J_{1}-J_{2}\right)
\end{array}\right\} \\
& f_{d}=0, \quad f_{\omega}=m g r_{0}\left\{\begin{array}{c}
e_{3} \cdot b_{2} \\
-e_{3} \cdot b_{1} \\
0
\end{array}\right\} \\
& \mathrm{d}=\lfloor x, y\rfloor^{T}, \dot{\omega}=\left\lfloor\begin{array}{lll}
\omega_{1} & \omega_{2} & \omega_{3}
\end{array}\right\rfloor^{T}, \lambda=\left\lfloor\begin{array}{lll}
\lambda_{1} & \lambda_{2} & \lambda_{3}
\end{array}\right\rfloor \\
& S(\mathbf{u})=0, \quad \mathbf{f}=\mathbf{0} \\
& \mathbf{b}=\mathbf{R e} \\
& \boldsymbol{\omega}=2\left(q_{0} \mathbf{g}-\tilde{\mathbf{g}} \dot{\mathbf{g}}-\dot{q}_{0} \mathbf{g}\right), \quad \mathbf{g}=\left\lfloor\begin{array}{lll}
q_{1} & q_{2} & q_{3}
\end{array}\right]^{\mathbf{T}}
\end{aligned}
$$

There is a total of eight variables in the foregoing equations of motion as given by (7.6). However, in adopting the present solution procedure-viz, (6.4)-(6.6)-we solve for nine variables.

Numerical solutions of the rolling of sphere on a flat sinusoidal curve have been obtained with the data summarized in Table 1. 
Table 1 Physical Dimensions and Initial Conditions for a Rolling Sphere

$$
\begin{gathered}
m=71.32 N, \quad a=10.9 \mathrm{~cm}, \quad r_{0}=0 \text { or } 0.15 \mathrm{~cm} \\
J_{1}=J_{2}=J_{3}=2 / 5 m a^{2}, \epsilon=10^{-6} \\
x^{0}=y^{0}=0, \quad\left\{\omega_{2}^{0}=-\omega_{1}^{0}=1, \omega_{3}^{0}=0\right\} \\
\dot{x}^{0}=\dot{y}^{0}=a \omega_{1}^{0}, \quad\left\{q_{0}^{0}=1, q_{1}^{0}=q_{2}^{0}=q_{3}^{0}=0\right\}
\end{gathered}
$$

The ball track curve on the flat surface with time is shown in Fig. 2 for the case of no offset $\left(r_{0}=0\right)$ and the corresponding angular velocities in Fig. 3. The time histories of the three constraint forces are shown in Fig. 4 wherein $\left(\lambda_{1}, \lambda_{2}\right)$ correspond to the $x$ and $y$ - component of the constraint force to maintain the rolling contact condition, and $\lambda_{3}$ is to maintain the sinusoidal trajectory as imposed by (7.1). Hence, the first two constraints are indicative of skidding phenomenon and the third corresponds to the steering force required in the ball manuevering. Notice that they exhibit highly nonlinear behavior while still periodic.

The ball track projected on the ball itself is shown in Figs. 5 through 8 , the $(x-z)$-plane view in Fig. 5, the $(y-z)$-plane view in Fig. 6, the $(x-y)$-plane view in Fig. 7 and a three-dimensional trajectory in Fig. 8.

Convergence studies have been performed with increasing stepsizes and it has been found that the present procedure, viz, (6.4)-(6.6), maintains both the solution accuracy and stability for the stepsize up to $h \leq 0.15$. Although not described herein, an implicit algorithm was tried out to solve the translational motions, $d$, thus replacing (6.4) by a corresponding implicit procedure. It was determined that, while larger stepsizes were permitted, we not only had to iterate to convergence at each time step but also required more computations due to matrix solutions at each step and/or iteration. We hope that 
further studies will illuminate how one can profit by a combined use of explicit and implicit algorithms to solve the translational part of the equations of motion.

Figures 9 - 11 show the $y$-direction surface traction of the ball with the offset center $\left(r_{0}=0.15 a\right)$, the angular velocities and the contraint forces. Note that, for the ball with no offset center, the average velocity in the $y$-direction is found from Fig. 2 to be about $1.2 \mathrm{unit} / \mathrm{sec}$. On the other hand, the corresponding average velocity with the offset center is about 0.35 unit/sec. from Fig. 9 or about $1 / 3$ of the no-offset case. A more dramatic variation with the offset center ball is illustrated in its angular velocities as shown in Fig. 10. Note that the angular velocities no longer exhibit periodic response, whereas they are periodic for the no-offset case ( see Fig. 3). Likewise, the steering force to follow the sinusoidal curve $(y=\sin x)$ becomes highly nonlinear, although nonlinearly periodic. The $x$ and $y$-direction contact force to maintain the rolling contact condition between the ball and the surface, although bounded, manifests extremely nonlinear behavior.

Our experience with the example problem indicates that the present computational procedure for handling large rotational motions coupled with translational motions is robust and efficient. It is important to note that the present procedure traces not only the angular motions accurately but more importantly the constraint forces and the four Euler parameters (although these are not presented here). We hope to test the present computational procedure for larger and flexible structural systems in the coming months and report further results.

\section{Concluding Remarks}

A coputational procedure for an accurate and efficient solution of large angular motions has been presented. The present procedure treats each nodal angular orientation separately in terms of the four-parameter Euler formula. Hence, one deals with only $(4 \times 4)$-matrices whose inversions are done explicitly. It has been found from our numerical experiments 
that it is essential to enforce the constraint condition on the Euler parameters (5.4) and its time derivatives at each integration step. The present procedure is thus well suited to a partitioned solution procedure wherein the translational motions are integrated separately from that of the rotations motions. In addition, the present procedure interfaces easily with the constraint force solution package as discussed in detail in Park and Chiou[14,15].

From a theoretical viewpoint, the present procedure can be considered a nonlinearly implicit procedure since the angular accelerations and angular velocities are used as agruments in the implicit solution matrices. The stability of the present procedure is at present difficult to assess and needs to be addressed in order for the procedure to take full advantage of its implicit characteristic. This stability question will require an involved analytical study and we hope to address this issue in the coming months.

\section{Acknowledgements}

The work reported herein was supported by NASA/Langley Research Center under Grant NAG - 1 - 756. The authors wish to thank Drs. Jerry Housner and Jeff Stroud for their keen interest and encouragement during the course of the present work. 


\section{References}

1. Hooker, W. and Margulies, G., "The Dynamical Attitude Equations for an N-body Satellite," J. Astronautical Science, 12, 123-128 (1965).

2. Roberson, R. and Wittenburg, J., "A Dynamical Formalism for an Arbitrary Number of Interconnected Rigid Bodies with Reference to the Problem of Satellite Attitude Control," Proc. the Third Int. Congress of Automatic Control, Butterworth, London, 1965.

3. Likins, P., “Analytical Dynamics and Nonrigid Spacecraft Simulation," Technical Report 32-1593, Jet Propulsion Laboratory, Pasadena, Ca., 1974.

4. Ho, J., "The Direct Path Method for Deriving the Dynamic Equations of Motion of a Multibody Flexible Spacecraft with Topological Tree Configuraton," AIAA Paper No. 74-786, AIAA Conference on Mechanics and Control of Flight, Anaheim, Calif., 1974.

5. De Veubeke, B. F., "The Dynamics of Flexible Bodies," Int. J. Engng. Sci., 14, 1976, 895-913.

6. Kane, T. and Levinson, D., "Formulation of Equâtioñ of Miótioñ for Complex Spacecraft," J. Guidance and Control, 3, 1980, 99-112.

7. Walton, W. C. and Steeves, E. C., "A new matrix theorem and its application for establishing independent coordinates for complex dynamical systems with constraints," NASA TR-R326, 1969.

8. Baumgarte, J. W., "Stabilization of constraints and integrals of motion in dynamical systems," Comp. Meth. Appl. Mech. Engr., 1, 1-16 (1972).

9. Baumgarte, J. W., "A New Method of Stabilization for Holonomic Constraints," Journal of Applied Mechanics, 50, 869-870 (1983).

10. Lötstedt, P., "On a penalty function method for the simulation of mechanical systems subject to constraints," TRITA-NA-7919, 1979, Royal Institute of Technology, Stockholm, Sweden.

11. Wehage, R. A. and Haug, E. J., "Generalized coordinate partitioning for dimension reduction in analysis of constrained dynamic systems," ASME J. of Mech. Design, 104, 1982, 247-255.

12. Huston, R. L. and Kamman, J. W., "A discussion on constraint equations in multibody dynamics," Mech. Res. Comm., 9, (1982) 251-256.

13. Fuehrer, C. and Wallrapp, O., "A computer-oriented method for reducing linearized multibody system equations by incorporating constraints," Comp. Meth. Appl. Mech. Engr., 46, (1984) 169-175. 
14. Park, K. C., "Stabilization of Computational Procedures for Constrained Dynamical Systems: Formulation," Presented at the 27th SDM Conf., San Antonio, Texas, May 1986, Paper No. AIAA-86-0926-CP.

15. Park, K. C. and Chiou, J. C., "Evaluation of Constraint Stabilization Procedures for Multibody Dynamical Systems," Proc. the 28th Structures, Structural Dynamics and Materials Conf., Part 2A, 1987, Monterey, CA, AIAA Paper No. 87-0927, 769-773.

16. Bodley, C. S., Devers, A. D., Park, A. C. and Frish, H. P., "A Digital Computer Program for the Dynamic Interaction Simulation of Controls and Structures (DISCOS)," NASA Technical Paper 1219, May 1978.

17. Orlandea, N., Chase, M. A. and Calahan, D. A., "A sparsity-oriented approach to the dynamic analysis and design of mechanical systems - part I and II," Trans. ASME, J. Eng. for Industry, Ser. B, 99, 1977, 773-784.

18. Park, K. C., Felippa, C. A. and DeRuntz, J. A., "Stabilization of staggered solution procedures for fluid-structure interaction analysis, " in: Computational Methods for Fluid-Structure Interaction Problems, Belytschko, T. and Geers, T. L. (editors), ASME, AMD Vol. 26, New York, N. Y., 1977, 95-124.

19. Park, K. C., "Partitioned Analysis Procedures for Coupled-Field Problems: Stability Analysis," Journal of Applied Mechanics, 47, 1980, 370-378.

20. Park, K. C. and Felippa, C. A., "Partitioned Analysis of Coupled Systems," in: Computational Methods for Transient Analysis, T. Belytschko and T. J. R. Hughes (eds.), Elsevier Pub. Co., 1983, 157-219.

21. Wittenburg, J., Dynamics of Systems of Rigid Bodies, B. G. Teubner, Stuttgart, 1977.

22. Huston, R. L., Passerello, C., Winget, J. M. and Sears, J., "On the Dynamics of a Weighted Bowling Ball," Journal of Applied Mechanics, 46, 1979, 937-943. 


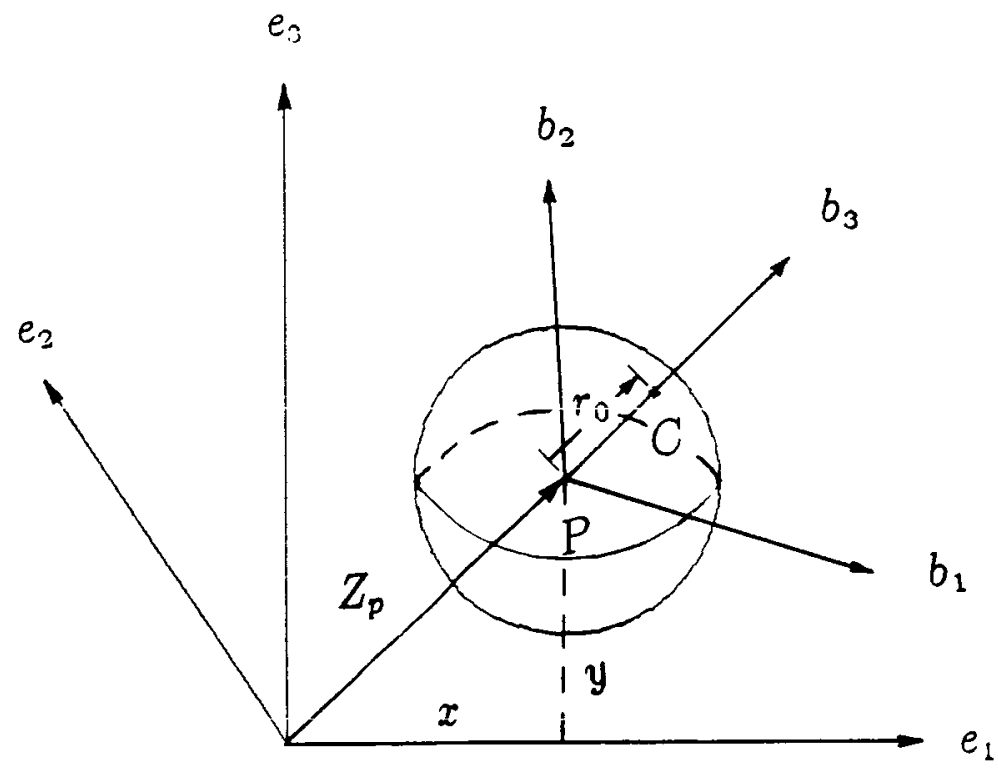

Fig. 1 Solid spherical ball rolling on a flat surface 


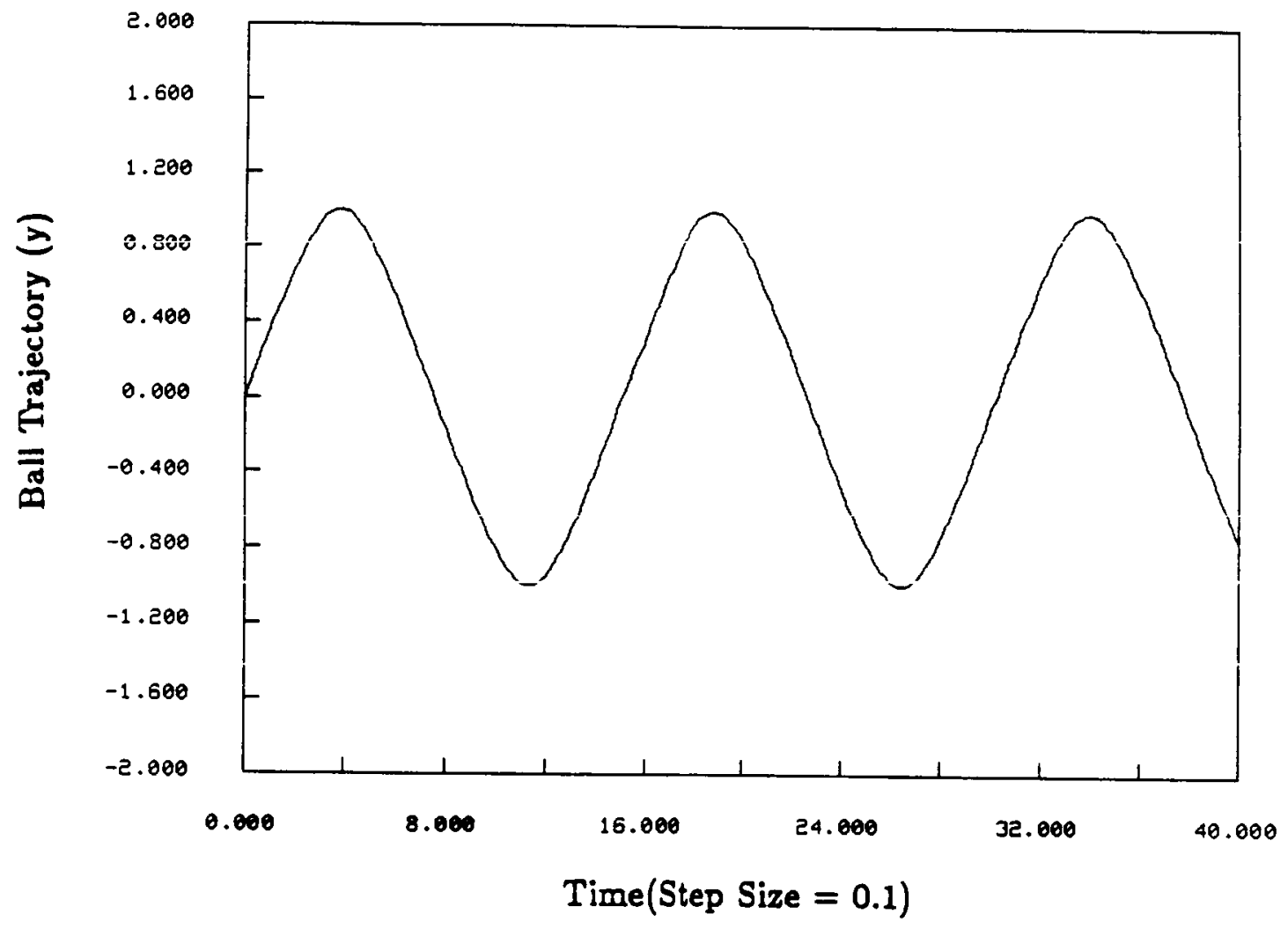

Fig. 2 Ball track on the flat surface with no offset 


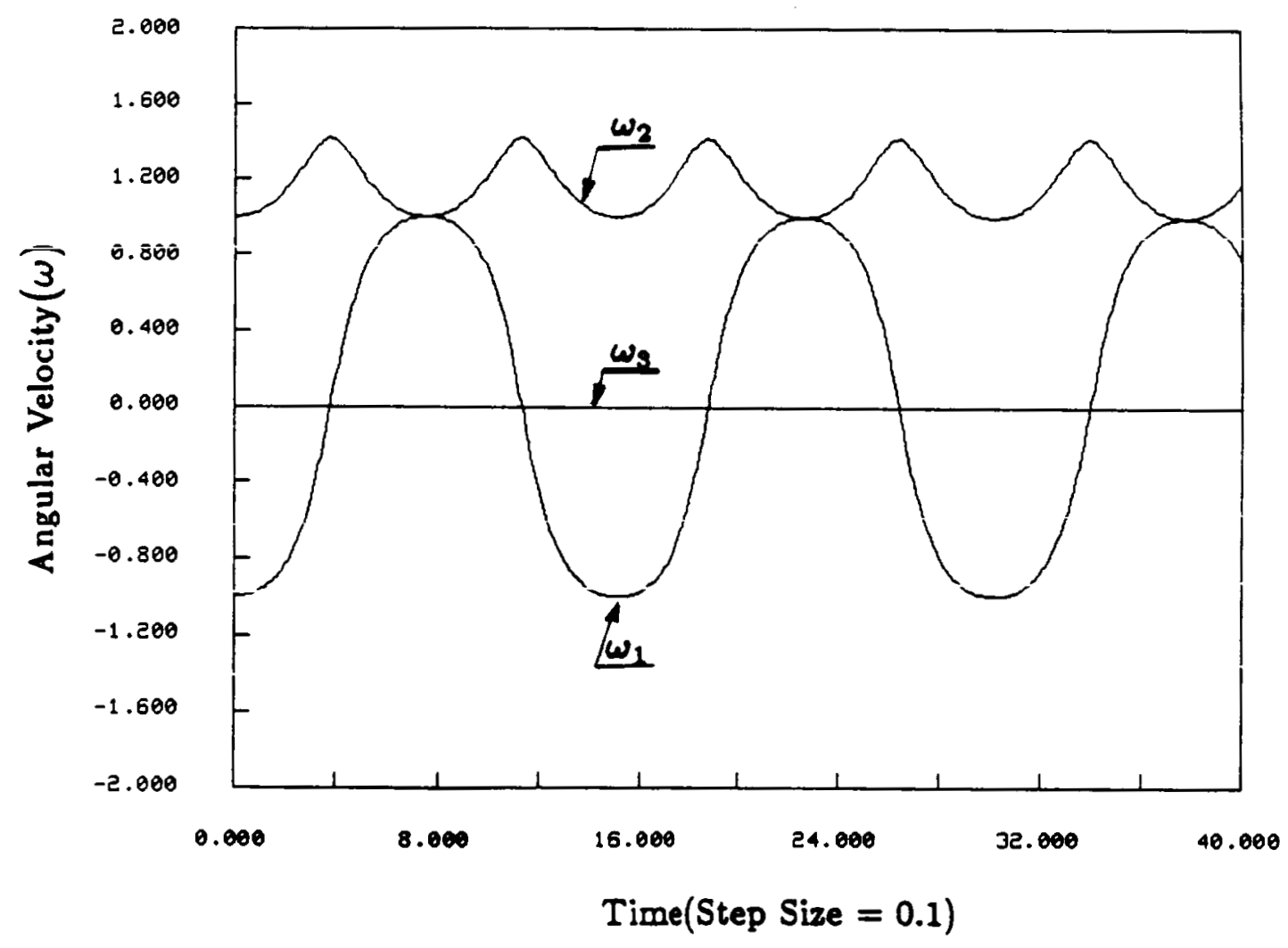

Fig. 3 Angular velocities of the sphere with no offset 


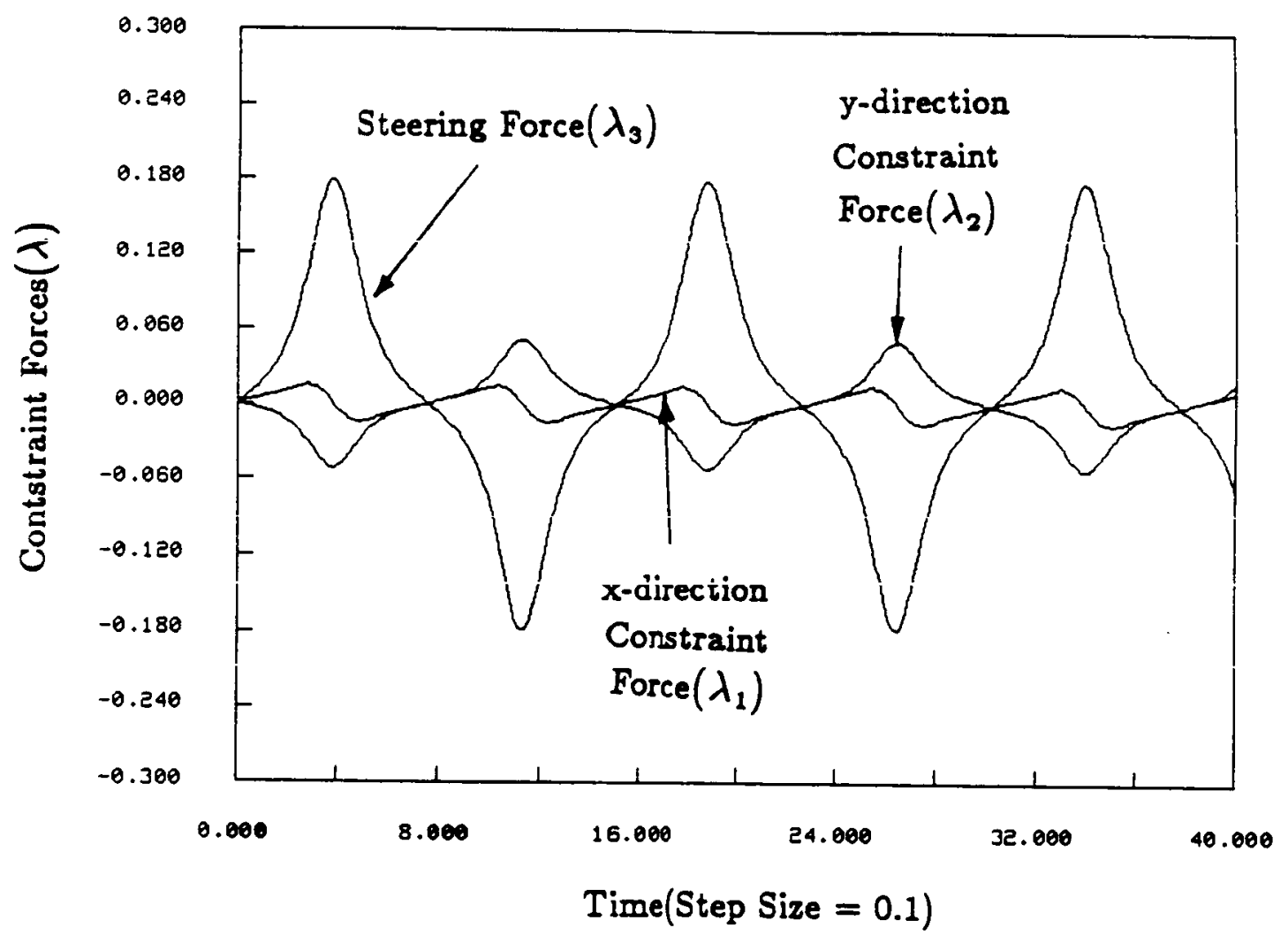

Fig. 4 Histories of three constraint forces on the rolling sphere 


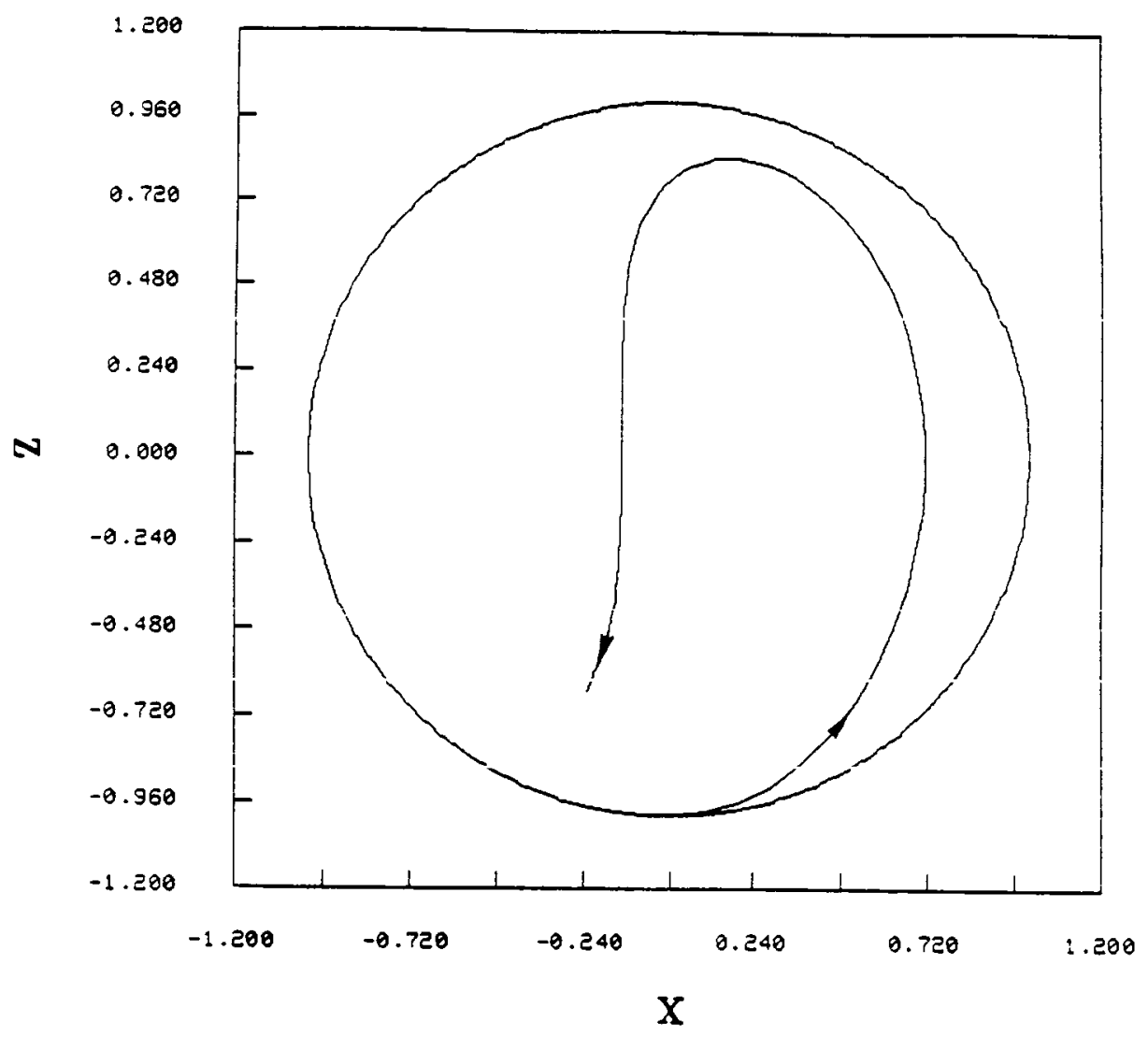

Fig. 5 Ball track projected on the $\mathrm{X}$ - Z plane of sphere 


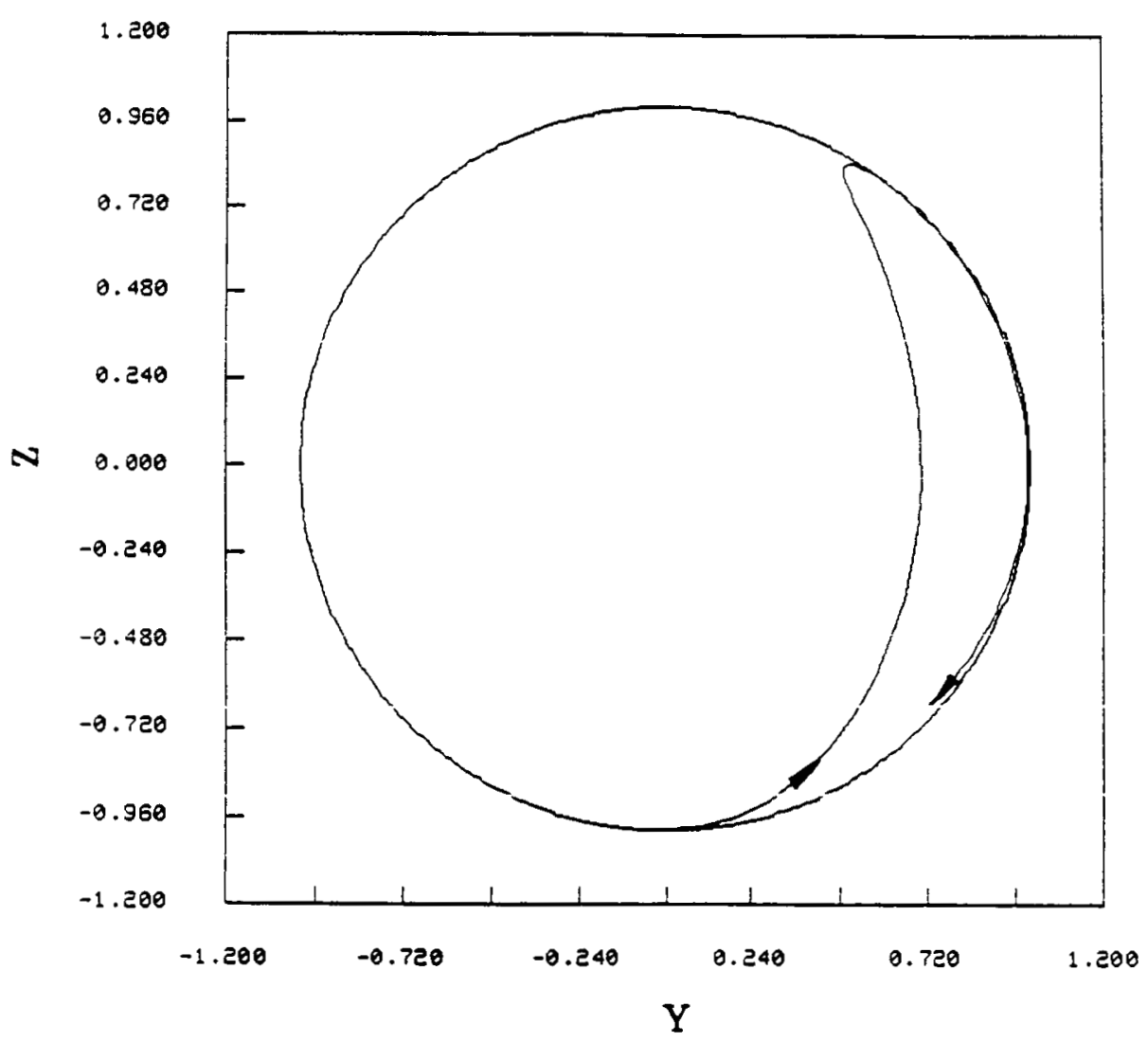

Fig. 6 Ball track projected on the $Y-Z$ plane of sphere 


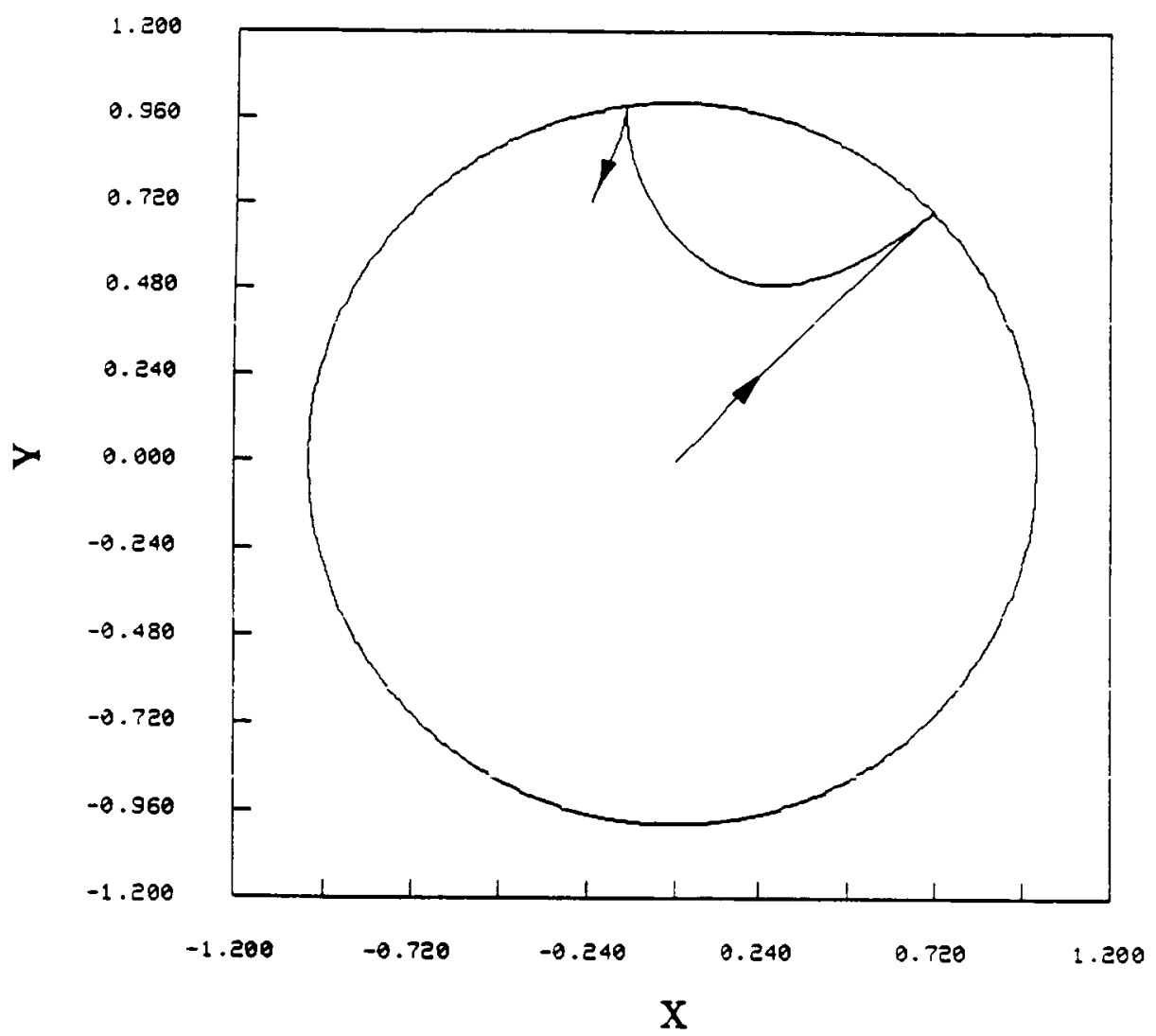

Fig. 7 Ball track projected on the $\mathrm{X}$ - Y plane of sphere 


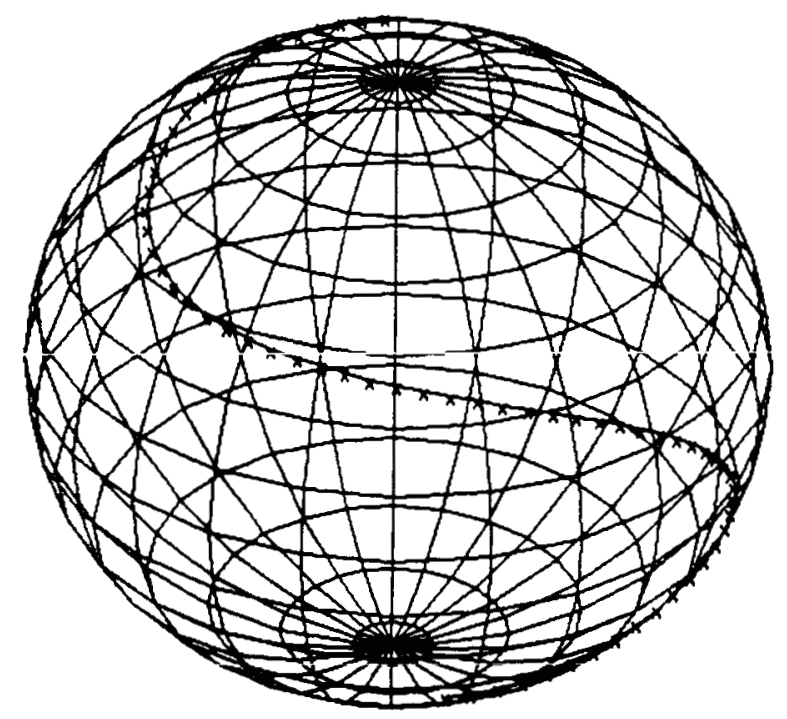

Fig. 8 Ball track projected on 3-D sphere surface 


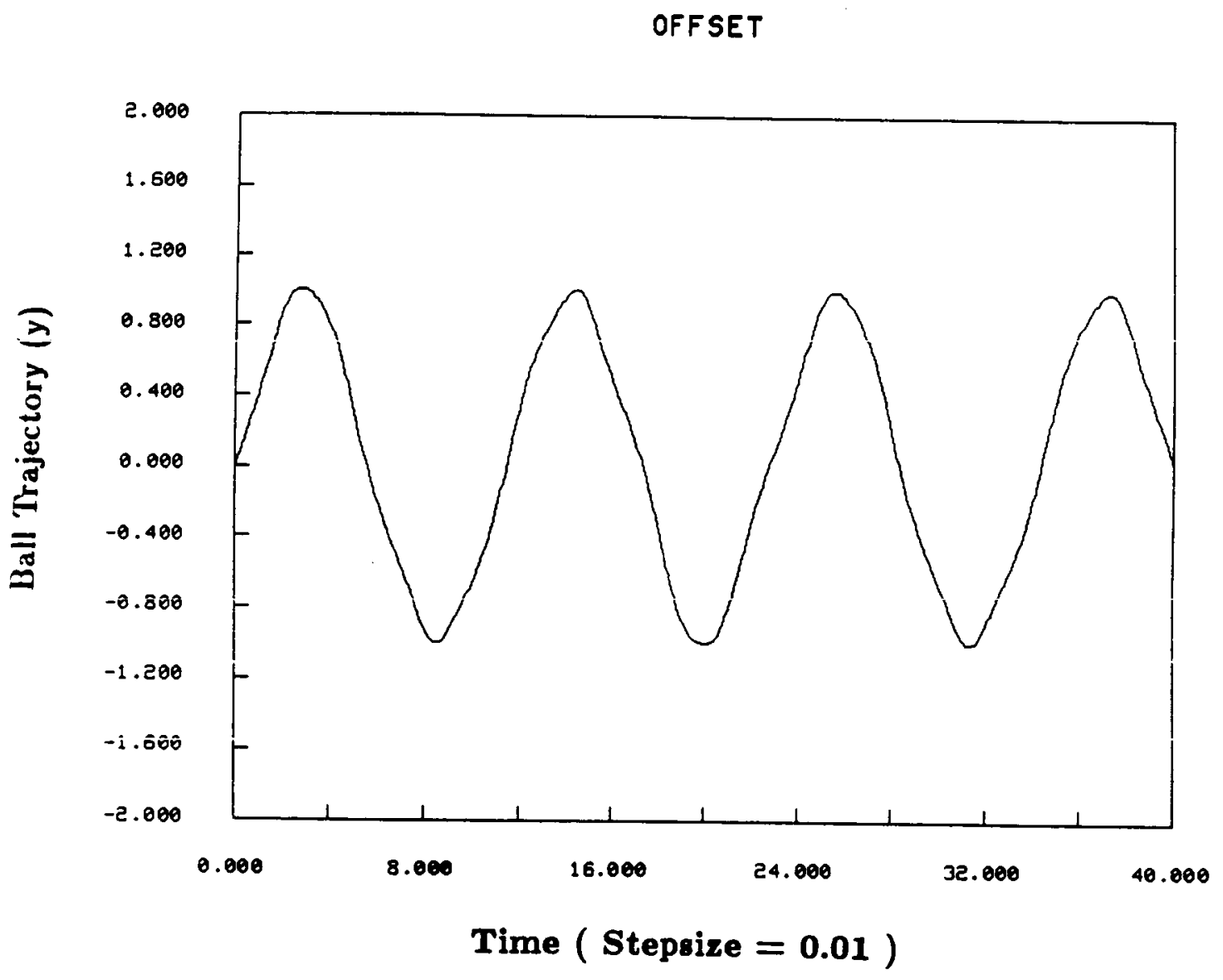

Fig. 9 Ball track on the flat surface with offset $\left(r_{0}=0.15 a\right)$ 


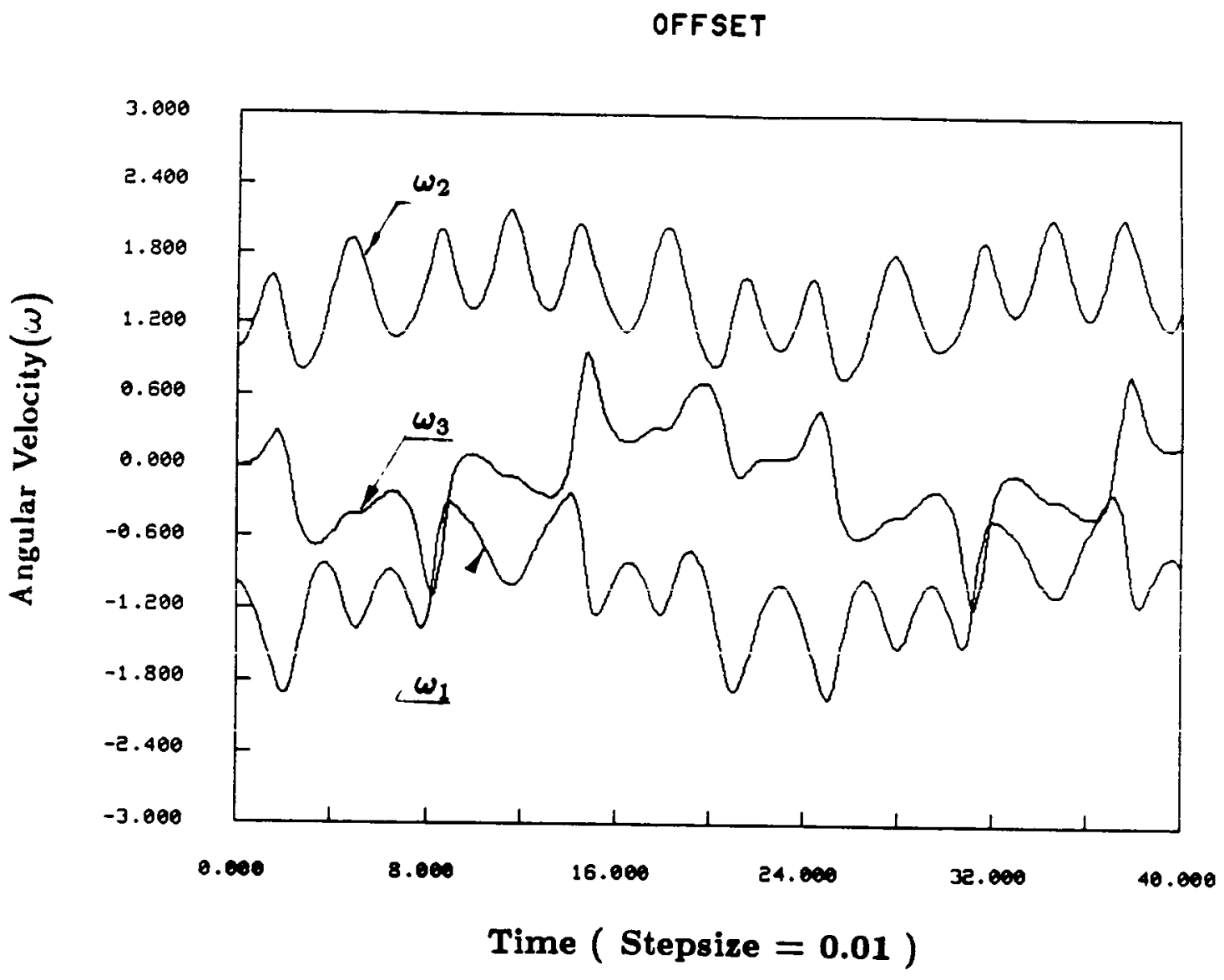

Fig. 10 Angular velocities of the sphere with offet center. 
OFFSET

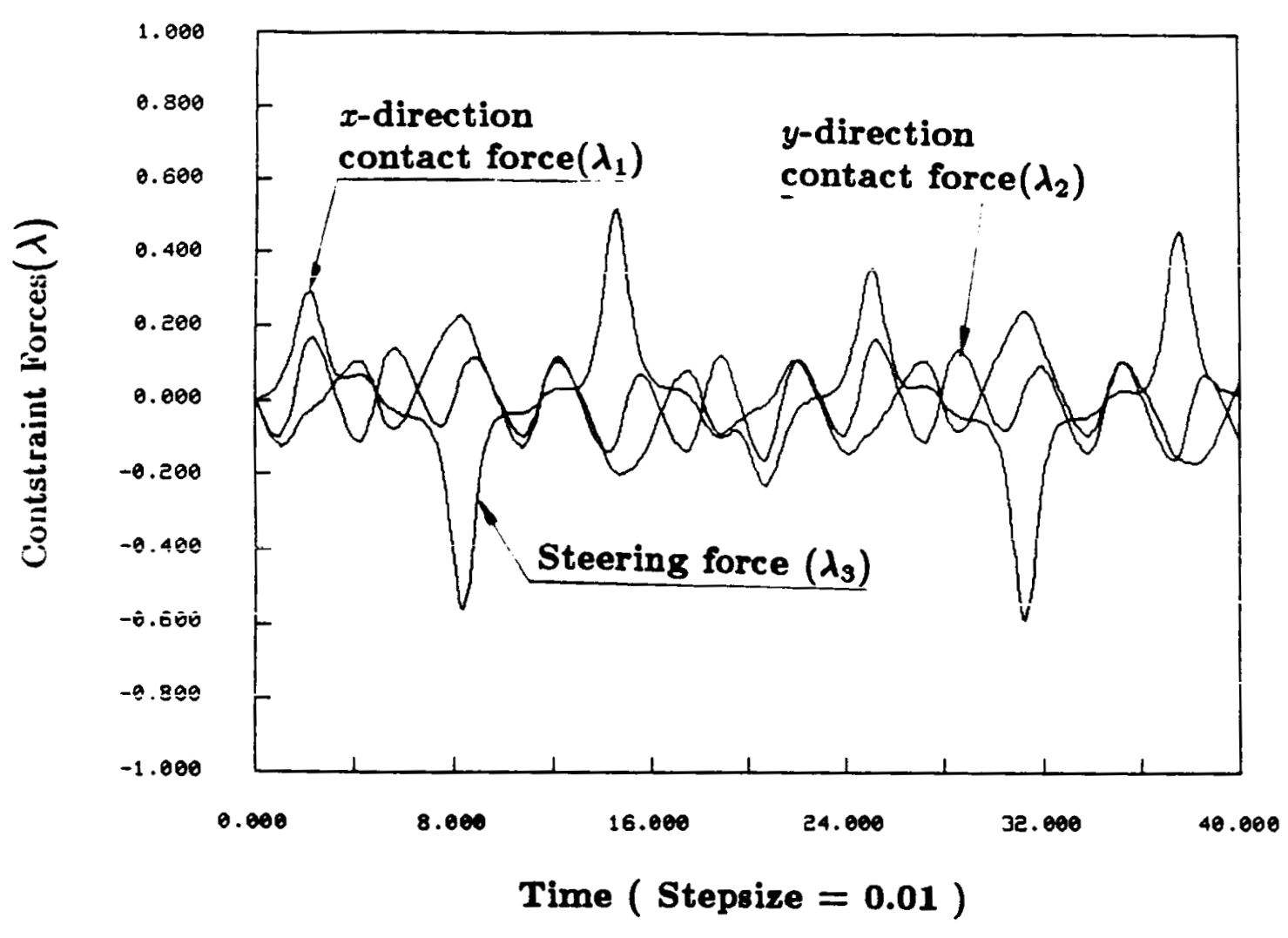

Fig. 11 Time histories of constraint forces with offet center 